\title{
Performance of Ad Hoc Networks with Two-Hop Relay Routing and Limited Packet Lifetime*
}

\author{
Ahmad Al Hanbali, Philippe Nain, and Eitan \\ INRIA, \\ B.P. 93, 06902 Sophia Antipolis Cedex, France \\ \{aalhanba,nain,altman\}@sophia.inria.fr
}

\begin{abstract}
Considered is a mobile ad hoc network consisting of three types of nodes (source, destination and relay nodes) and using the two-hop relay routing protocol. Packets at relay nodes are assumed to have a limited lifetime in the network. All nodes are moving inside a bounded region according to some random mobility model. Both closed-form expressions, and asymptotic results when the number of nodes is large, are provided for the packet delivery delay and the energy needed to transmit a packet from the source to its destination. We also introduce and evaluate a variant of the two-hop relay protocol that limits the number of generated copies in the network. Our model is validated through simulations for two mobility models (random waypoint and random direction mobility models), numerical results for the two-hop relay protocols are reported, and the performance of the two-hop routing and of the epidemic routing protocols are compared.
\end{abstract}

\section{Keywords}

Mobile Ad hoc network; Delay Tolerant Network; Two-hop relay protocol; Limited packet lifetime; Mobility model; Performance analysis.

\section{INTRODUCTION}

Ad hoc Networks are complex distributed systems, that are composed of wireless mobile or static nodes that can freely and dynamically self-organize. In this way they form arbitrary, and temporary "ad hoc" network topologies, allowing devices to seamlessly interconnect in areas with no pre-existing infrastructure.

In a Mobile Ad Hoc Network (MANET), since there is no fixed infrastructure and nodes are mobile, links between nodes are set up and turn down dynamically. A link be-

\footnotetext{
${ }^{*}$ The authors acknowledge the support of the European IST project BIONETS and of the Network of Excellence (NoE) EuroNGI.
}

Permission to make digital or hard copies of all or part of this work for personal or classroom use is granted without fee provided that copies are not made or distributed for profit or commercial advantage and that copies bear this notice and the full citation on the first page. To copy otherwise, to republish, to post on servers or to redistribute to lists, requires prior specific permission and/or a fee.

Valuetools'06 , October 11-13, 2006, Pisa, Italy.

Copyright 2006 ACM 1-59593-504-5 /06/10...\$5.00 tween two nodes is up when these nodes are inside one another communication range, and a link is down otherwise. The establishment of a route from a source node to a destination node requires the simultaneous availability of a number of links that are all up, one originating at the source node and another one ending at the destination nodes. Indeed, MANETs often experience route failures and network disconnectivity, especially when the nodes are moving frequently and the network is sparse. Grossglauser and Tse [9] have observed that mobility in MANETs can be used to increase the average network throughput. Their idea was to look at the diversity gain achieved by using the mobile nodes as relays. Their relay mechanism, called two-hop relay protocol, is simple: if there is no route between the source node and the destination node, the source node transmits its packets to all neighboring nodes (called relay nodes) that it meets for delivery to the destination. A relay node is only allowed to send a packet to its destination node, and it is not allowed to send the packet to another relay node, thereby justifying the name of this protocol. It was then shown in [6] that a bounded delay can be guaranteed under this relaying mechanism. The aim of these studies (see also [10]) is the scaling property of the throughput or delay as the number of nodes in the network becomes large. Our interest in the present work is in the performance of the above mentioned relaying mechanism in a network consisting of a fixed finite number of nodes.

It is important to mention that most of the studies of scaling laws of delay and throughput in wireless MANETs assume a uniform spatial distribution of nodes, which is the case, for example, when the nodes perform a symmetric Random Walk over the region of interest $[6,9]$, or when nodes move according to the Random Direction model [13]. In the present work, we replace this assumption by assuming that the inter-meeting time between two nodes, defined as the time duration between two consecutive points in time where these nodes meet (i.e. come within transmission range of one another), is exponentially distributed. The validity of this assumption has been discussed in [8], and its accuracy has been shown for a number of mobility models (Random Walker, Random Direction, Random Waypoint) in the case when the node transmission range is small with respect to the area where the nodes evolve. It is worth pointing out that for some of the mobility models (non-symmetric Random Walk and Random Waypoint) nodes are not uniformly distributed over the area of interest.

The type of mobile networks that we address in this paper belongs to Delay Tolerant Network (DTN) [1] in which the 
incurred delay to send data between nodes can be very large and unpredictable. In our case this high delay is due to the high node mobility, low node density, and short node transmission range. Hence in these cases, most of the time the network is disconnected, and there are no routes between nodes. For this reason we assume that the transfer of data between nodes is done through the relay nodes using the two-hop relay protocol.

The objective of this paper is to study a number of performance metrics bearing on the packet delivery delay and the overhead induced by the two-hop relay protocol (see Section 2 ). This will be done under the assumption that, unlike in $[8,15]$, packets at relay nodes have a limited lifetime in the network.

Another relay protocol closely related to the two-hop relay protocol is the so-called epidemic routing protocol [14, 16]. This protocol is identical to the two-hop relay protocol, except that in the epidemic routing protocol a relay node is allowed to transmit a packet to any node that its meets, including another relay node. Epidemic routing decreases the delivery delay of packets at the cost of increasing the energy consumption by the network. The performance of both the two-hop relay protocol and the epidemic routing protocol will also be compared in this paper.

The rest of the paper is organized as follows: Section 2 gives a careful description of the two-hop relay protocol, sets the modeling assumptions, and defines the performance metrics of interest (delivery delay, overhead in terms of the number of copies of a packet). In Section 3 we develop a Markovian analysis that yields closed-form expressions for these performance metrics. In Section 4, we propose and evaluate a modification of the two-hop relay protocol, called $K$-limited two-hop relay protocol, that aims at limiting the overall energy consumption. Section 5 presents an asymptotic analysis of the performance metrics as the number of nodes is large; this analysis uses a mean-field approximation. Validation of our model, and comparison of the performance of the two-hop relay protocol and the epidemic routing protocol are given in Section 6. Section 7 concludes the paper and suggests some research directions.

\section{THE SYSTEM MODEL}

We consider the model introduced in [8]. In this model the characteristics of MANETs are captured through a single parameter, $1 / \lambda$, representing the expected inter-meeting time between any pair of nodes. More precisely, there are $N+1$ nodes consisting of: one source node, one destination node, and $N-1$ relay nodes. Two nodes may only communicate at certain points in time, called meeting times. The time that elapses between two consecutive meeting times of a given pair of nodes is called the inter-meeting time. In [8] it is assumed that inter-meeting times are mutually independent and identically distributed (iid) random variables (rvs), with an exponential distribution with intensity $\lambda>0$.

Throughout this paper we address the scenario where the source node wants to send a single packet to the destination node. To this end the source may use the relay nodes, as explained below. In this paper, we will focus on the two-hop relay protocol [9].

The two-hop relay protocol works as follows. The source node keeps sending a copy of the packet to all nodes that it meets and that do not have a copy, including the destination node, until the destination node has received a copy of the packet. Transmissions between two nodes are assumed to be instantaneous. This corresponds to the situation where the transfer time of a packet between two nodes is negligible with respect to their inter-meeting time. The way the source node is notified that the destination node has received the packet, either directly from it or from a relay node, is irrelevant for the metrics that we will consider (see below). A relay node that possesses a copy of the packet is only allowed to send it to the destination node, thereby justifying the name of this protocol (two-hop relay protocol).

In addition to the model in [8] we assume throughout this paper that each copy of the packet has a Time-To-Live (TTL). When the TTL of a copy expires then the copy is destroyed. TTLs are assumed to be iid rvs with an exponential distribution with rate $\mu>0$. The packet to be sent by the source has no TTL associated with it, so that the source is always able to send a copy to another node (if the packet at the source has a TTL then there is a non-zero probability that the destination node will never receive the packet. This scenario is not considered in this paper).

What are the results obtained in our simple setting (single packet and instantaneous transmission times) that could shed light on the performance of the two-hop relay protocol in more realistic contexts (multiple packets, non-zero transmission times, limited relay storage capacity, etc.)? First, note that the packet delivery delay obtained in our setting gives a lower-bound, as a consequence of the instantaneous transmission time. Second, the protocol overhead, measured in terms of the total number of copies per-packet generated, gives an upper-bound. This is so because in the realistic context the source will not systematically be able to transmit a packet to a relay node that it encounters.

We assume that the source is ready to transmit the packet to the destination at time $t=0$. The (packet) delivery time (or delivery delay), $T_{d}$, is the first time after $t=0$ when the destination node receives the packet (or a copy of the packet).

In the following we will investigate the delivery delay, the number of copies in the system at the delivery time, and the total number of copies generated by the source before the delivery time (Section 3). The latter is related to the overhead induced by the two-hop relay protocol and, in particular, to the total energy needed to transmit the packet to the destination (Section 4).

A word on the notation: throughout $\mathbf{1}_{A}$ will designate the indicator function of any event $A\left(\mathbf{1}_{A}=1\right.$ if $A$ is true and 0 otherwise) and $\operatorname{diag}\left(a_{1}, \ldots, a_{n}\right)$ will define a $n$-by- $n$ diagonal matrix with $(i, i)$-entry $a_{i}$.

\section{MARKOVIAN ANALYSIS}

The state of the system is represented by the random variable $I(t) \in\{1,2, \ldots, N, a\}$, where $I(t) \in\{1,2, \ldots, N\}$ gives the number of copies when the packet has not been delivered to the destination (i.e. for $0 \leq t<T_{d}$ ) and $I(t)=a$ for $t \geq T_{d}$. Under the assumptions made in Section 2, $\{I(t), \bar{t} \geq 0\}$ is an absorbing, finite-state, continuous-time Markov chain, with transient states $\{1,2, \ldots, N\}$ and absorbing state $a$. Let $\mathbf{P}=[p(i, j)]$ be the one-step transition matrix of the absorbing, finite-state, discrete-time Markov chain (referred to as $\mathbf{M C}$ from now on) embedded just before the jump times of the Markov chain $\{I(t), t \geq 0\}$. From the transition rate diagram of Markov chain $\{I(t), t \geq 0\}$ in 
Figure 1 we find

$$
\begin{aligned}
p(i, i+1) & =\frac{(N-i) \rho}{N \rho+i-1}, \quad i=1, \ldots, N-1, \\
p(i, i-1) & =\frac{i-1}{N \rho+i-1}, \quad i=2, \ldots, N, \\
p(i, a) & =\frac{i \rho}{N \rho+i-1}, \quad i=1, \ldots, N \\
p(i, j) & =0, \text { otherwise, }
\end{aligned}
$$

with $\rho:=\lambda / \mu$.

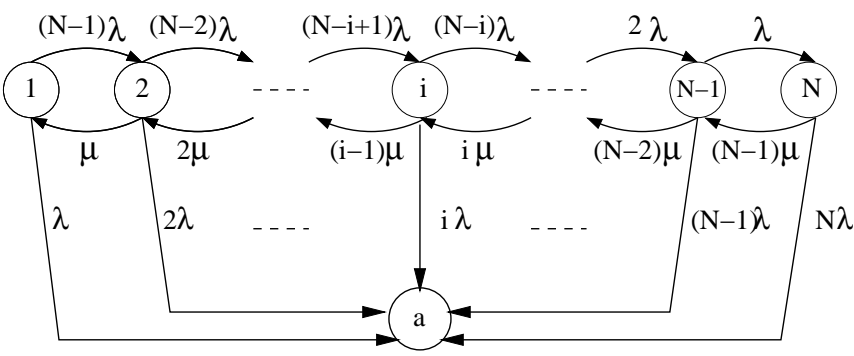

Figure 1: Transition rate diagram of the Markov chain $\{I(t), t \geq 0\}$.

The transition matrix $\mathbf{P}$ of the Markov chain $\mathbf{M C}$ can be written as

$$
\mathbf{P}=\left(\begin{array}{c|c}
\mathbf{Q} & \mathbf{R} \\
\hline \mathbf{0} & 1
\end{array}\right)
$$

where $\mathbf{Q}=[p(i, j)]_{1 \leq i, j \leq N}, \mathbf{R}=(p(1, a), \ldots, p(N, a))^{T}$, and $\mathbf{0}$ is the row vector of dimension $N$ whose all components are equal to 0 .

Define $\mathbf{M}=(\mathbf{I}-\mathbf{Q})^{-\mathbf{1}}$, the fundamental matrix of the absorbing Markov chain MC. The $(i, j)$-entry of $\mathbf{M}$, denoted by $m(i, j)$, gives the expected number of visits to state $j$ given that $I(0)=i[7$, Chap. 11, Theorem 11.4]. The matrix $\mathbf{M}$ is computed in explicit form in Lemma 1 in Appendix I, and $m(i, j)$ is given in (16).

We are now in position to compute the expected delivery delay, the distribution of the number of copies at the delivery instant, and the expected number of copies generated by the source.

\subsection{Delivery delay}

In this section we first determine $E_{i}\left[T_{d}\right]$, the expected delivery delay given that $I(0)=i \in\{1,2, \ldots, N\}$, from which the expected delivery delay $\overline{T_{d}}=E_{1}\left[T_{d}\right]$ will follow.

$E_{i}\left[T_{d}\right]$ is the expected time before absorption starting from the transient state $i$. Let $n_{i j}$ be the number of visits to state $j$ before absorption given that the chain starts in state $i$, and let $T_{j l}$ be the sojourn time in state $j$ at the $l$ th visit to that state. Observe that $E\left[n_{i j}\right]=m(i, j)$, where $m(i, j)$ is given in Lemma 1 , and that $E\left[T_{j l}\right]=1 /(N \lambda+\mu(j-1))$ for $j=1,2, \ldots, N$ (see Figure 1). Hence,

$$
E_{i}\left[T_{d}\right]=\sum_{j=1}^{N} E\left[\sum_{l=1}^{n_{i j}} T_{j l}\right]=\sum_{j=1}^{N} m(i, j) E\left[T_{j l}\right],
$$

where the last equality follows from Wald's identity, since $n_{i, j}$ is independent of the rvs $\left\{T_{j l}\right\}_{j, l}$. Plugging the value found in (16) for $m(i, j)$ in the latter equation gives

$$
E_{i}\left[T_{d}\right]=-\frac{1}{\mu}\left(\left(\begin{array}{c}
N-1 \\
i-1
\end{array}\right) \rho^{i-1}\right)^{-1} \sum_{k=1}^{N} \frac{\boldsymbol{\Psi}^{k} \mathbf{1}^{T}}{z_{k} \boldsymbol{\Psi}^{k} \tau^{2}\left(\mathbf{\Psi}^{k}\right)^{T}} \Psi_{i}^{k},
$$

with $\mathbf{1}^{T}$ the $N$-dimensional column vector whose all components are equal to 1 . Quantities $\Psi_{i}^{k}, \tau$ and $z_{k}$ are defined in Lemma 1 in Appendix I. Note that these quantities are only dependent on $\rho$ and $N$.

More generally, the tail probability distribution of $T_{d}$, starting from $I(0)=i$ is given by

$$
P_{i}\left(T_{d} \geq t\right)=\frac{1}{\left(\begin{array}{c}
N-1 \\
i-1
\end{array}\right) \rho^{i-1}} \sum_{k=1}^{N} \frac{\boldsymbol{\Psi}^{k} \mathbf{1}^{T}}{\boldsymbol{\Psi}^{k} \tau^{2}\left(\mathbf{\Psi}^{k}\right)^{T}} \Psi_{i}^{k} e^{z_{k} \mu t} .
$$

The proof of this result is shown in Appendix II. Observe that $P_{i}\left(T_{d} \geq t\right)$ is nothing than a weighted sum of exponentials of weights and exponents that depend $\rho, \mu$, and $N$.

\subsection{Number of copies in the system at delivery time}

Let $P_{i}\left[C_{d}=j\right]$ be the probability that the number of copies in the network at the delivery time is $j$, given there are $i$ copies in the network at time $t=0$. We assume without loss of generality that the Markov chain $\mathbf{M C}$ is leftcontinuous so that $P_{i}\left[C_{d}=j\right]=P\left[I\left(T_{d}-\right)=j\right]$ (by convention $I(t-)$ is the state of the process $\mathbf{M C}$ just before time $t)$. In words, $P_{i}\left[C_{d}=j\right]$ is the probability that the last visited state before absorption is $j$, given that the initial state is $i$.

If we split the absorbing state $a$ into $N$ absorbing states $a_{1}, \ldots, a_{N}$, as shown in Figure 2, we will not affect the dynamics of the original Markov chain before absorption. This means that the fundamental matrix of the modified absorbed Markov chain is the same as the fundamental matrix of the original absorbed Markov chain. Clearly, $P_{i}\left[C_{d}=j\right]$ is now equal to the probability that the modified chain is absorbed in state $a_{j}$. Let $b_{i, a_{j}}$ denote this probability. From the theory of absorbing Markov chains, we see that [7, Chap. 11, Theorem 11.6]

$$
b_{i, a_{j}}=\sum_{k=1}^{N} m(i, k) r\left(k, a_{j}\right),
$$

where $r\left(k, a_{j}\right)$ is the one-step transition probability from state $k$ to the absorbing state $a_{j}$ in the modified Markov chain. Clearly (see Figure 2) $r\left(k, a_{j}\right)=j \lambda /(N \lambda+(j-1) \mu)=$ $j \rho /(N \rho+j-1)$ if $k=j$ and $r\left(k, a_{j}\right)=0$ if $k \neq j$. Therefore,

$$
P_{i}\left[C_{d}=j\right]=m(i, j) r\left(j, a_{j}\right)
$$

The $n$ th-order moment of $C_{d}$ is equal to

$$
E_{i}\left[C_{d}^{n}\right]=-\frac{1}{\left(\begin{array}{c}
N-1 \\
i-1
\end{array}\right) \rho^{i-2}} \sum_{k=1}^{N} \frac{\Psi_{i}^{k}}{z_{k} \Psi^{k} \tau^{2}\left(\Psi^{k}\right)^{T}} \Psi^{k} \mathbf{J}_{n+1}^{T},
$$

with $\mathbf{J}_{n}:=\left(1, \cdots, i^{n}, \cdots, N^{n}\right)$. Coming back to the original problem, the probability distribution of the number of copies at delivery time is given by $P_{1}\left[C_{d}=j\right]$, and the $n$-th order moment is given by $E_{1}\left[C_{d}^{n}\right]$.

\subsection{Total number of copies generated by the source before delivery time}

The objective is to find, $\overline{G_{d}}$, the expected number of copies generated by the source before the delivery time (or equivalently, before absorption). Let $G_{d}^{i, j}$ be the number of copies 


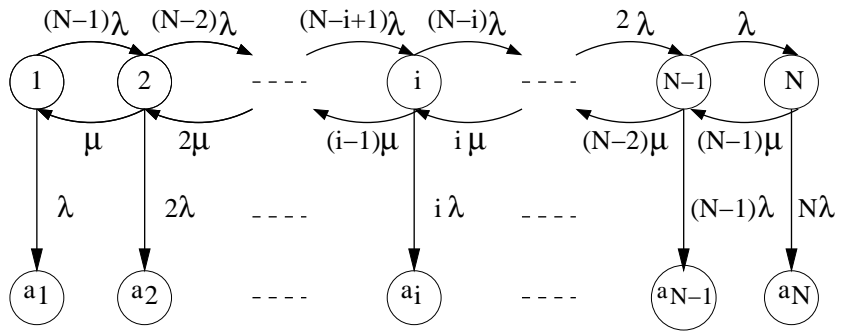

Figure 2: The modified absorbing Markov chain with $N$ absorbing states.

generated by the source before absorption given that the chain starts in state $i$ and that state $j$ is the last state visited before absorption (i.e. $I\left(T_{d}-\right)=j$ ). Introduce $J^{i, j}(k, k+1)$ (resp. $\left.J^{i, j}(k+1, k)\right)$ the number of transitions from state $k$ (resp. state $k+1$ ) to state $k+1$ (resp. state $k$ ) given that $I(0)=i$ and $I\left(T_{d}-\right)=j$. It is easy to see that for $k=1,2, \ldots, N-1$ that

$$
J^{i, j}(k, k+1)=J^{i, j}(k+1, k)+\mathbf{1}_{\{i \leq k<j\}}-\mathbf{1}_{\{j \leq k<i\}},
$$

A copy of the packet is generated by the source each time there is a transition from state $k$ to state $k+1$ for all states $k=1,2, \ldots, N-1$. Hence,

$$
G_{d}^{i, j}=\sum_{k=1}^{N-1} J^{i, j}(k, k+1) .
$$

On the other hand, $n_{i, k}^{j}$, the total number of visits to state $k$ given that $I(0)=i$ and $I\left(T_{d}-\right)=j$, satisfies the relation

$$
\sum_{k=1}^{N} n_{i, k}^{j}=\sum_{k=1}^{N-1} J^{i, j}(k, k+1)+\sum_{k=1}^{N-1} J^{i, j}(k+1, k)+1 .
$$

From (4) we find that

$$
\sum_{k=1}^{N-1} J^{i, j}(k+1, k)=\sum_{k=1}^{N-1} J^{i, j}(k, k+1)+i-j .
$$

Combining the three last identities gives

$$
G_{d}^{i, j}=\frac{1}{2}\left[\sum_{k=1}^{N} n_{i, k}^{j}+j-i-1\right] .
$$

The expected number of copies given that $I(0)=i$, denoted by $\overline{G_{d}^{i}}$, is given by (Hint: remove the conditioning on $C_{d}=j$ )

$$
\overline{G_{d}^{i}}=\frac{1}{2}\left[\sum_{k=1}^{N} m(i, k)+E_{i}\left[C_{d}\right]-i-1\right]
$$

where $m(i, k)$ is given in Lemma 1 and $E_{i}\left[C_{d}\right]$ is given in (3) (with $n=1$ ). Finally, $\overline{G_{d}}=\overline{G_{d}^{1}}$. Note that the probability distribution of $G_{d}$ can be computed by defining a twodimensional and continuous-time absorbing Markov chain with state $(i, c)$, where $i \in\{1, \cdots, N\}$ represents the number of copies in the network, and $c \in \mathbf{N}$ denotes the total number of copies generated by the source. Thus, $P\left[G_{d}=l\right]$ is the probability that the absorption occurs at one of the following transient states $\{(i, l): 1 \leq i \leq \min (l+1, N)\}$.

We will see in the next section how $\overline{G_{d}}$ can be used to compute the overall energy needed to transmit a packet to the destination.

\section{LIMITED ENERGY CONSUMPTION}

We will only consider the energy consumption due to packet transmission and decoding. Let $p_{t}$ be the energy needed at the sender to transmit a packet to another node and let $p_{r}$ be the energy needed at the receiver to decode a packet. The energy consumed by the source before the packet is delivered to the destination $P_{s}=p_{t} \overline{G_{d}}$, since the source needs to generate on the average $\overline{G_{d}}$ copies of the packet before one copy reaches the destination. The energy consumed by all nodes before the delivery time is given by $P_{d}=\left(p_{t}+p_{d}\right) \overline{G_{d}}$.

In this section we introduce and evaluate a new two-hop relay scheme that limits the energy consumption by limiting the number of copies that the source can generate before the packet reaches the destination. A similar scheme was introduced in [15] to limit the energy consumption of epidemic routing. We now assume that the source can generated at most $K$ copies of the packet. In the following this scheme will be referred to as the $K$-limited two-hop relay protocol. Alike in the original protocol in Section 3 (corresponding to $K=\infty$ ), we will compute the expected delivery delay and the expected number of copies generated before the delivery time for the $K$-limited two-hop relay protocol. The behavior of the $K$-limited two-hop relay protocol can be modeled as a two-dimensional, finite-state, absorbing and continuoustime Markov chain (referred to as $\overline{\mathbf{M C}}_{K}$ ) with state $(i, c)$, where $i \in\{1,2, \ldots, N\}$ gives the number of copies in the network, and $c \in\{0,1, \ldots, K\}$ records the total number of copies generated by the source. It is easy to see that the one-step probability transition matrix $\mathbf{P}_{K}=\left[p_{K}((i, c), \cdot)\right]$ of the absorbing, finite-state, discrete-time Markov chain (referred to as $\left.\mathbf{M} \mathbf{C}_{K}\right)$ ) embedded just before the jump times of $\overline{\mathbf{M C}}_{K}$ is given by

$$
\begin{aligned}
& p_{K}((i, c),(i+1, c+1))=\frac{(N-i) \rho}{N \rho+(i-1)}, \quad 1 \leq i \leq K_{m} \text { and } \\
& p_{K}((i, c),(i-1, c)) \quad=\frac{(i-1)}{N \rho+(i-1)}, \quad 2 \leq i \leq K_{m} \text { and } \\
& p_{K}((i, c), a) \\
& p_{K}((N, c),(N-1, c))=\frac{(N-1)}{N \rho+(N-1)} \mathbf{1}_{\{K \geq N\}}, \\
& p_{K}((N, c), a) \\
& p_{K}((i, K),(i-1, K) \\
& p_{K}((i, K), a) \\
& p_{K}((i, c),(j, d)) \\
& i-1 \leq c \leq K-1 \text {, } \\
& =\frac{i \rho}{N \rho+(i-1)}, \quad 1 \leq i \leq K_{m} \text { and } \\
& i-1 \leq c \leq K-1 \text {, } \\
& \begin{array}{c}
N-1 \leq c \leq K-1, \\
=\frac{N \rho}{N \rho+(N-1)} \mathbf{1}_{\{K \geq N\}}, \\
N-1 \leq c \leq K-1,
\end{array} \\
& =\frac{(i-1)}{i \rho+(i-1)}, \quad 2 \leq i \leq K_{m}+1, \\
& =\frac{i \rho}{i \rho+(i-1)}, \quad 1 \leq i \leq K_{m}+1 \text {, } \\
& =0 \text {, otherwise, }
\end{aligned}
$$

with $K_{m}:=\min (K, N-1)$, and where $a$ is the absorbing state. Let $L$ denotes the total number of transient states. If $K \leq N-1$ then $L=L_{1}:=(K+1)(K+2) / 2$ whereas if $K>N$ then $L=L_{2}:=N(2 K-N+3) / 2$.

If we label the transient states $(1,0)$ as $1,(2,1)$ as $2, \cdots$ ,$(i, c)$ as $\frac{(c+1)(c+2)}{2}-i+1$ for $c \leq K_{m}$ and $i \leq c+1, \cdots$, $(i, c)$ as $\frac{N(2 c-N+1)}{2}+N-i+1$ for $c>K_{m}$ and $i \leq N$, then we can write the matrix $\mathbf{P}_{K}$ as

$$
\mathbf{P}_{K}=\left(\begin{array}{c|c}
\mathbf{Q}_{K} & \mathbf{R}_{K} \\
\hline \mathbf{0} & 1
\end{array}\right),
$$

where $\mathbf{Q}_{K}$ is an L-by-L matrix giving the one-step transition probability between two transient states, $\mathbf{R}_{K}$ is an L-by1 matrix giving the one-step transition probability from a 
transient state to the absorbing state $a$, and $\mathbf{0}$ is the 1 -by- $\mathrm{L}$ zero matrix.

The fundamental matrix associated with the absorbing Markov chain $\mathbf{M} \mathbf{C}_{K}$ is $\mathbf{M}_{K}=\left(\mathbf{I}-\mathbf{Q}_{K}\right)^{-1}$. Let $m_{K}(i, j)$ be the $(i, j)$-entry of $\mathbf{M}_{K}$. The matrix $\left(\mathbf{I}-\mathbf{Q}_{K}\right)$ can be seen as an upper-bidiagonal block matrix that it is easy to invert recursively. Once the matrix $\mathbf{M}_{K}$ has been computed the main performance metrics can easily be deduced, as shown below.

\subsection{Expected delivery delay}

We distinguish the cases $K \leq N-1$ and $K \geq N$. In the former case, the expected delivery delay given that the chain starts in state $(1,0)$ reads

$$
\begin{aligned}
\overline{T_{d}^{K}=} & \sum_{i=1}^{K+1} \frac{m_{K}\left(1, L_{1}-i+1\right)}{i \lambda+(i-1) \mu}+ \\
& \sum_{i=1}^{K} \frac{\sum_{j=i-1}^{K-1} m_{K}(1, a(i, j))}{N \lambda+(i-1) \mu},
\end{aligned}
$$

where $a(i, j):=1-i+\frac{(j+1)(j+2)}{2}$.

If $K \geq N$ we find

$$
\begin{gathered}
\overline{T_{d}^{K}}=\sum_{i=1}^{N} \frac{m_{K}\left(1, L_{2}-i+1\right)}{i \lambda+(i-1) \mu}+ \\
\sum_{i=1}^{N} \frac{\sum_{j=i-1}^{N-1} m_{K}(1, a(i, j))+\sum_{j=N}^{K-1} m_{K}(1, b(i, j))}{N \lambda+(i-1) \mu}
\end{gathered}
$$

where $b(i, j):=N-i+1+\frac{N(2 j-N+1)}{2}$.

\subsection{Expected number of copies}

The expected number of copies generated by the source before the delivery time, given that the chain starts in state $(1,0)$ is given by

$$
\overline{G_{d}^{K}}=\sum_{c=1}^{K} c \sum_{i=1}^{N_{c}} P(\text { absorption occurs in state }(i, c)),
$$

with $N_{c}:=\min (c+1, N)$.

If $K \leq N-1$ then

$$
\begin{aligned}
\overline{G_{d}^{K}}= & \rho \sum_{c=1}^{K-1} \sum_{i=1}^{c+1} i c \frac{m_{K}(1, a(i, c))}{N \rho+i-1}+ \\
& \rho K \sum_{i=1}^{K+1} i \frac{m_{K}(1, a(i, K))}{i \rho+i-1},
\end{aligned}
$$

while if $K \geq N$

$$
\begin{gathered}
\overline{G_{d}^{K}}=\rho \sum_{c=1}^{N-1} \sum_{i=1}^{c+1} i c \frac{m_{K}(1, a(i, c))}{N \rho+i-1}+ \\
\rho \sum_{c=N}^{K-1} \sum_{i=1}^{N} i c \frac{m_{K}(1, b(i, c))}{N \rho+i-1}+\rho K \sum_{i=1}^{N} i \frac{m_{K}(1, b(i, K))}{i \rho+i-1},
\end{gathered}
$$

where $a(i, c)$ and $b(i, c)$ are defined in Section 4.1.

The energy consumed by the source before the packet is delivered to the destination is given by $p_{t} \overline{G_{d}^{K}}$ while the energy consumed by all nodes during this period is $\left(p_{t}+\right.$ $\left.p_{d}\right) \overline{G_{d}^{K}}$.

\section{ASYMPTOTIC ANALYSIS}

In this section we derive asymptotic results for the expected delivery delay and the expected number of copies at delivery instant in the two-hop relay protocol when the number of nodes $N$ is large. Deriving these asymptotic results from the explicit formulas in (1) and (3), respectively, is not easy (in the more simpler case when there are no timeouts getting asymptotics from the explicit results were already quite involved [8, Appendix A]).

We shall instead follow a mean field approach to find approximations of these asymptotics. The same approach was used in [14] and in [16] to derive asymptotic results for epidemic models.

The mean field approximation says that $X(t)$ (resp. $G(t)$ ), the expected number of copies (resp. of copies generated by the source) in the network at time $t$, before absorption, when $N$ is large, can be approximated by the solution of the following 1st-order differential equation (see [12] for the general theory)

$$
\begin{aligned}
\dot{X}(t) & =\lambda(N-X(t))-\mu(X(t)-1), \quad t>0 . \\
\dot{G}(t) & =\lambda(N-X(t)), \quad t>0 .
\end{aligned}
$$

This equation simply reflects the fact that at time $t X(t)$ increases with the rate $\lambda(N-X(t))$ and decreases with the rate $\mu(X(t)-1)$. We need to complement this equation with another equation whose the solution approximates $D(t):=$ $P\left(T_{d}<t\right)$, the probability distribution of the delivery delay. It was found in [14] that

$$
\dot{D}(t)=\lambda X(t)(1-D(t)), \quad t>0 .
$$

Solving (9), (10), and (11) with the initial conditions $X(0)=$ $x_{0}\left(x_{0}=1\right.$ in our model $), G(0)=0$, and $D(0)=0$ yields

$$
\begin{aligned}
X(t) & =\frac{N \lambda+\mu}{\lambda+\mu}+\left(x_{0}-\frac{N \lambda+\mu}{\lambda+\mu}\right) e^{-(\lambda+\mu) t} \\
G(t) & =\lambda N t-f_{N}(t), \quad D(t)=1-e^{-f_{N}(t)}
\end{aligned}
$$

where $f_{N}(t):=\frac{\lambda}{\lambda+\mu}\left[(N \lambda+\mu) t+\left(x_{0}-\frac{N \lambda+\mu}{\lambda+\mu}\right)\left(1-e^{-(\lambda+\mu) t}\right)\right]$. It can be checked that $D(0)=0, \lim _{t \rightarrow \infty} D(t)=1$ and $t \rightarrow D(t)$ is nondecreasing, so that $D(t)$ is indeed a probability distribution of a proper rv. As expected from the very definition of $X(t)$, we note that $X(\infty)=(N \lambda+\mu) /(\lambda+\mu)$ is the expected stationary number of customers in a finitestate birth and death process, with birth rate (resp. death rate) $\lambda(N-i)($ resp. $\mu(i-1))$ in state $i \in\{1,2, \ldots, N\}$.

\subsection{Delivery delay}

By definition, $E\left[T_{d}\right]=\int_{0}^{+\infty} P\left(T_{d}>t\right) d t$, so that from (12) $E\left[T_{d}\right]$ can be approximated by

$$
E\left[T_{d}\right] \approx \int_{0}^{+\infty} e^{-f_{N}(t)} d t
$$

when $N$ is large. When $N$ is large it is easily seen that the dominant contribution of $e^{-f_{N}(t)}$ to the above integral comes from small values of $t$ since $f_{N}(t)$ is a nondecreasing function of $N$. Hence, $e^{-f_{N}(t)}$ can be approximated by $e^{-f_{N}^{\prime \prime}(0) t^{2} / 2}$ since $f_{N}(0)=0$ and since $f_{N}^{\prime}(0)=\lambda x_{0}$ does not depend on $N$, with $f_{N}^{\prime \prime}(0)=\lambda\left(N \lambda+\mu-(\lambda+\mu) x_{0}\right)$. For $0 \leq x_{0}<X(\infty)$ this gives the 1st-order asymptotics

$$
E\left[T_{d}\right] \approx \sqrt{\frac{\pi}{2 \lambda\left(N \lambda+\mu-(\lambda+\mu) x_{0}\right)}} \approx \frac{1}{\lambda} \sqrt{\frac{\pi}{2 N}}
$$


for $N \rightarrow \infty$. The 2nd-order asymptotics of $E\left[T_{d}\right]$ can be obtained by expanding $f_{N}(t)$ in Taylor series at the order three in the vicinity of $t=0$. We find

$$
\begin{aligned}
E\left[T_{d}\right] \approx & \int_{0}^{+\infty} e^{-\frac{f_{N}^{\prime \prime}(0)}{2 !} t^{2}}\left(1-\frac{f_{N}^{(3)}(0)}{3 !} t^{3}\right) d t \\
= & \sqrt{\frac{\pi}{2 \lambda\left(N \lambda+\mu-(\lambda+\mu) x_{0}\right)}}+ \\
& \frac{(\lambda+\mu)\left(N \lambda+\mu-(\lambda+\mu) x_{0}\right)}{3 \lambda^{3}(N-1)^{2}}
\end{aligned}
$$

for $N \rightarrow \infty$. Asymptotics, as $N$ is large, for any order moment of $T_{d}$ can be derived using a similar approach (see [11]).

Figure 3 displays the 1st-order and 2nd-order asymptotics of $E\left[T_{d}\right]$, given in (14) and in (15), respectively, as a function of $N$, and compare them with the exact value obtained in (1). We observe that, as $N$ increases, both asymptotics converge to the exact result.

\subsection{Expected number of copies at delivery in- stant}

When $N$ is large, $E\left[C_{d}\right]$, the mean number of copies at the delivery time $T_{d}$, is approximated by $\int_{0}^{+\infty} X(t) d D(t)$. With the use of (12) an integration by part gives

$$
E\left[C_{d}\right] \approx x_{0}+\left(N \lambda+\mu-(\lambda+\mu) x_{0}\right) \int_{0}^{\infty} e^{-f_{N}(t)-(\lambda+\mu) t} d t
$$

for $N \rightarrow \infty$. By using again the property that the dominant contribution of $e^{-f_{N}(t)-(\lambda+\mu) t}$ to the above integral comes from small values of $t$. we may approximate $e^{-f_{N}(t)-(\lambda+\mu) t}$ by $e^{-f_{N}^{\prime \prime}(0) t^{2} / 2}$. Hence,

$$
E\left[C_{d}\right] \approx x_{0}+\sqrt{\frac{\pi}{2 \lambda}} \sqrt{N \lambda+\mu-(\lambda+\mu) x_{0}} \approx \sqrt{\frac{\pi N}{2}}
$$

for $N \rightarrow \infty$.

\subsection{Expected number of copies}

When $N$ is large, $E\left[G_{d}\right]$, the mean number of copies generated by the source before delivery time $T_{d}$, is approximated by $\int_{0}^{+\infty} G(t) d D(t)$. With the use of (13) an integration by part gives

$$
E\left[G_{d}\right] \approx \lambda N \int_{0}^{+\infty} e^{-f_{N}(t)} d t-1 \approx \sqrt{\frac{\pi N}{2}} \quad(N \rightarrow \infty) .
$$

From these asymptotic results of $E\left[T_{d}\right]$ and $E\left[G_{d}\right]$ we derive the Little-like formula $E\left[G_{d}\right] \approx \lambda N E\left[T_{d}\right](N \uparrow \infty)$ relating the total expected number of copies generated by the protocol (protocol overhead) to the expected delivery time.

\section{NUMERICAL RESULTS}

In this section we first validate the Markov model introduced in Section 3 by comparing its performance (expected delivery delay) to that obtained by simulations, for two different mobility models (Random Waypoint (RWP) and Random Direction (RD) models). Simulation results of RWP and $\mathrm{RD}$ are obtained using the NS-2 code of the random trip model [4]. We then compare the expected delivery delay and the energy consumption induced by the two-hop relay protocol and the epidemic protocol. We conclude by investigating the performance of the $K$-limited two-hop relay protocol.

\subsection{Model validation}

We have simulated the two-hop relay protocol with exponential timeouts for both the RWP and the RD mobility models. In the RWP model [5] each node is assigned an initial location in a given area (typically a square) and travels at a constant speed to a destination chosen randomly in this area. The speed is chosen randomly in $\left(v_{\min }, v_{\max }\right)$, independently of the initial location and destination. After reaching the destination the node may pause for a random time, after which a new destination and speed are chosen, independently of previous speeds, destinations, and pause times. In the RD model [3] each node is assigned an initial direction, speed and travel time. The node then travels in that direction at the given speed and for the given duration. When the travel time has expired, the node may pause for a random time, after which a new direction, speed and travel time are chosen at random, independently of all previous directions, speeds and travel times. When a node reaches a boundary it is either reflected or the area wraps around so that the node reappears on the other side. In both mobility models nodes move independently of each other.

In our simulation settings, for both the RWP and the $\mathrm{RD}$ models the area is a square of side-length $L=2000 \mathrm{~m}$, the speed is constant and equals to $V=10 \mathrm{~m} / \mathrm{sec}$., there is no pause time, and the transmission range $R$ is constant and the same for all nodes. In addition, in the RD model the travel time is constant and equals to $30 \mathrm{sec}$. and the nodes reflect on reaching the boundaries. It has been experimentally observed in [8] that whenever $R<<L$ then the node inter-meeting time is exponentially distributed with rate $\lambda=10.94 \frac{R V}{\pi L^{2}}$ for RWP and $\lambda=8 \frac{R V}{\pi L^{2}}$ for RD.

For different values of the ratio $R / L$ and for different values of the number of nodes $N$, Table 1 reports the expected delivery delay obtained from the exact result in (1) and by simulations for the RWP model, and give relative errors. The same results for the RD model are reported in Table 2.

From the results in Tables 1-A, 2-A we conclude that, for both mobility models, our model is accurate for $N$ relatively small. Observe that more accurate results are reported for the $\mathrm{RD}$ model for a given ratio $R / L$ and a given number of nodes. In addition, the relative error as a function of $R / L$ was evaluated and our model shows good results for small value of $R / L$ especially in the case of $\mathrm{RD}$ model, see Table 2-B.

\subsection{Comparison of two-hop and epidemic routing protocols}

In this section, we compare the expected delivery delay, $E\left[T_{d}\right]$, and the expected number of packets transmitted, $E\left[G_{d}\right]$, as a function of $\mu$, the timeout intensity, for the twohop relay and the epidemic routing protocols. The absorbing Markov chain modeling the epidemic routing protocol is the same as the absorbing Markov chain in Section 3, except that the birth rate in state $i$ is now equal to $\lambda i(N-i)$, since in the epidemic routing protocol all nodes are allowed to generate copies of the packet. The death rate (resp. absorption rate ) in state $i$ is unchanged and equal to $\mu(i-1)$ (resp. $\lambda i$ ). The computation of the expected delivery delay and the expected number of packets transmitted for the epidemic routing protocol is therefore similar to that carried out for the two-hop relay protocol, except that the fundamental matrix for the epidemic routing protocol cannot be computed in explicit form. This matrix was obtained nu- 


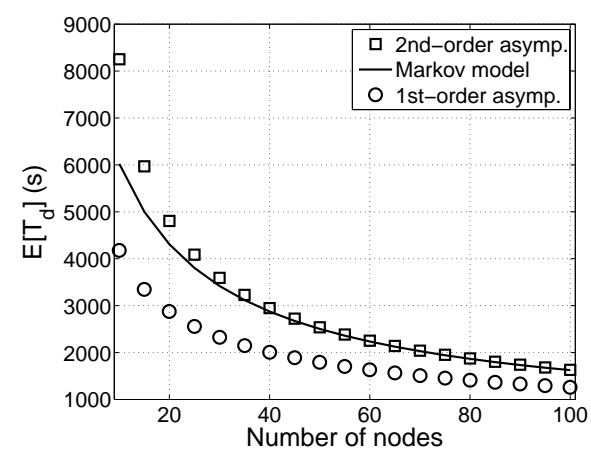

(a)

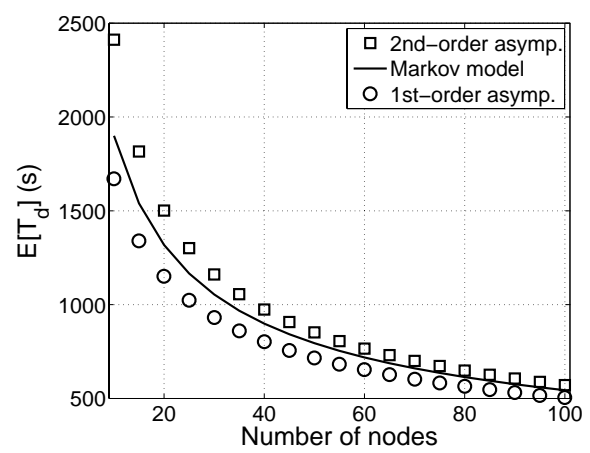

(b)

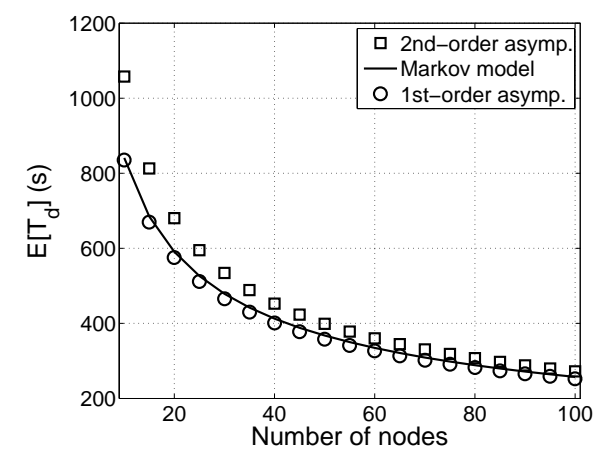

(c)

Figure 3: Comparing asymptotics for the expected delivery delay to the exact result $(\mu=0.001:(\mathrm{a}) \lambda=0.0001$, (b) $\lambda=0.00025$, (c) $\lambda=0.0005$.)

\begin{tabular}{|l|l|l|l|l|}
\hline$R / L(\%)$ & 1.25 & 1 & 0.5 & 0.1 \\
\hline$E_{m}\left[T_{d}\right](\mathrm{s})$ & 1216 & 1529 & 3154 & 20102 \\
\hline$E_{\text {sim }}\left[T_{d}\right](\mathrm{s})$ & 945 & 1245 & 2851 & 20861 \\
\hline$\left|1-\frac{E_{\text {Sim }}\left[T_{d}\right]}{E_{m}\left[T_{d}\right]}\right|(\%)$ & 22 & 18 & 10 & 4 \\
\hline
\end{tabular}

(A)

\begin{tabular}{|l|l|l|l|l|l|}
\hline$N$ & 10 & 20 & 30 & 40 & 100 \\
\hline$E_{m}\left[T_{d}\right](\mathrm{s})$ & 4344 & 3154 & 2596 & 2257 & 1436 \\
\hline$E_{\text {sim }}\left[T_{d}\right](\mathrm{s})$ & 4093 & 2851 & 2237 & 1839 & 1068 \\
\hline$\left|1-\frac{E_{\text {Sim }}\left[T_{d}\right]}{E_{m}\left[T_{d}\right]}\right|(\%)$ & 6 & 9 & 14 & 18 & 26 \\
\hline
\end{tabular}

(B)

Table 1: Expected delivery delay calculated from (1) and by simulations for the RWP model $(\mu=0.0001:(A)$ $N=20$ and $R / L$ decreases. (B) $R / L=0.5 \%$ and $N$ increases).

merically.

As expected, we observe that the epidemic routing protocol induces a smaller expected delivery delay than the twohop relay protocol, but at the expense of a much more important overhead in terms of the number of copies generated Figures 4 and 5. We also point out that the conclusions drawn from the results in Figure 5 apply for the energy consumptions $P_{s}$ and $P_{d}$ in the case where the energy to transmit (resp. decode) a packet is constant, since we have shown in Section 4 that in this case $P_{s}$ and $P_{d}$ are both linear functions of $E\left[G_{d}\right]$.

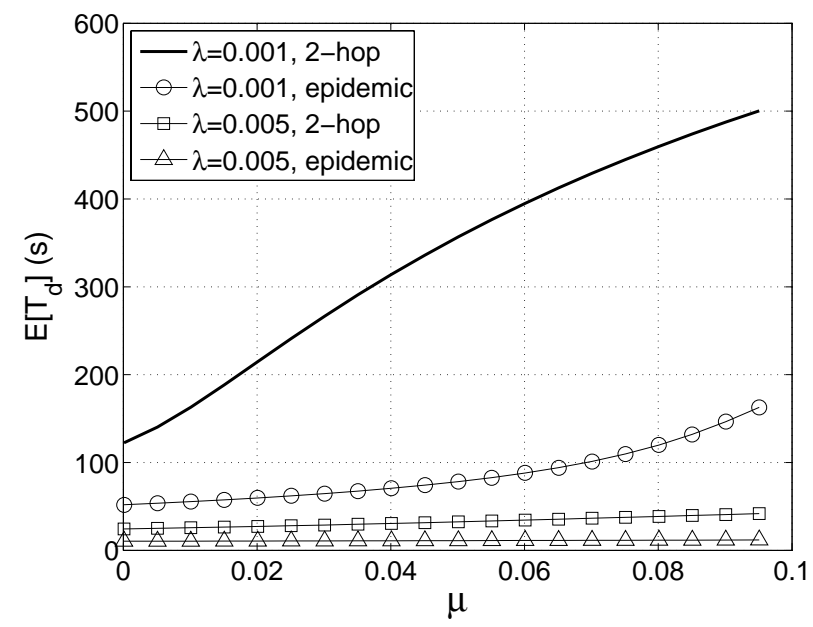

Figure 4: Expected delivery delay for two-hop relay and epidemic routing protocols as a function of $\mu$ $(N=100)$.

\subsection{Limited energy consumption}

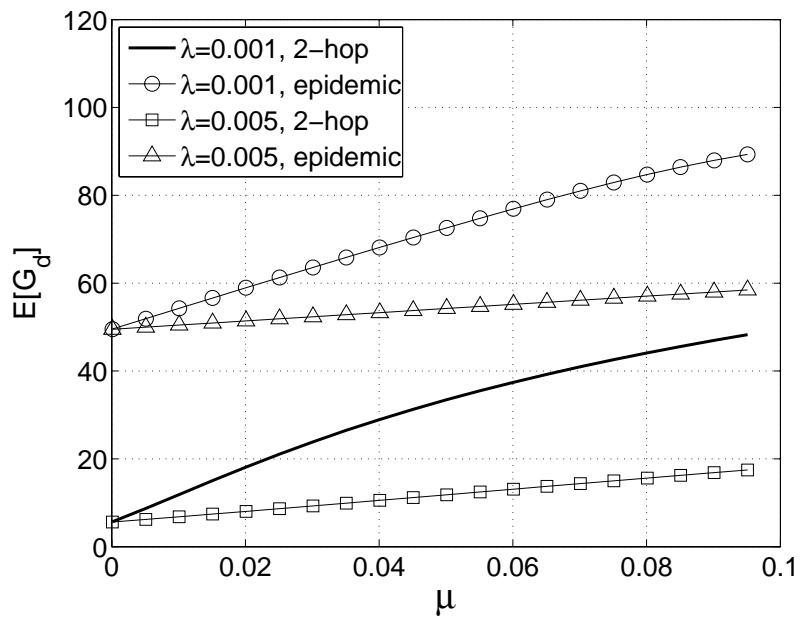

Figure 5: Expected number of packet transmitted for two-hop relay and epidemic routing protocols as a function of $\mu(N=100)$.

For different values of $\lambda$, the inter-meeting time rate, Figure 6 plots the expected delivery time, $\overline{T_{d}^{K}}$, under the $K$ limited two-hop relay protocol for different values of $K$, the maximum number of copies of the packet that the source may generate (see Section 4). For each $\lambda$, we observe there exists a threshold $K_{0}$ such that $\overline{T_{d}^{K}}$ is almost constant when $K \geq K_{0}\left(K_{0} \sim 20\right.$ for $\left.\lambda=0.001\right)$. Also, this constant value of $\overline{T_{d}^{K}}$ when $K \geq K_{0}$ is nothing than the mean delivery delay obtained in (1) of the original two-hop relay protocol

\section{CONCLUDING REMARKS}

In this work, we have evaluated the main performance metrics of the two-hop relay protocol under the assumption that packets in relay nodes have a limited lifetime. Closed- 


\begin{tabular}{|l|l|l|l|l|}
\hline$R / L(\%)$ & 1.25 & 1 & 0.5 & 0.1 \\
\hline$E_{m}\left[T_{d}\right](\mathrm{s})$ & 1678 & 2116 & 4416 & 30264 \\
\hline$E_{\text {sim }}\left[T_{d}\right](\mathrm{s})$ & 1596 & 1988 & 4176 & 31651 \\
\hline$\left|1-\frac{E_{s i m}\left\lfloor T_{d}\right]}{E_{m}\left\lceil T_{d}\right\rfloor}\right|(\%)$ & 6 & 6 & 5 & 4 \\
\hline
\end{tabular}

\begin{tabular}{|l|l|l|l|l|l|}
\hline$N$ & 10 & 20 & 30 & 40 & 100 \\
\hline$E_{m}\left[T_{d}\right](\mathrm{s})$ & 6116 & 4416 & 3622 & 3141 & 1987 \\
\hline$E_{\text {sim }}\left[T_{d}\right](\mathrm{s})$ & 6208 & 4141 & 3297 & 2867 & 1512 \\
\hline$\left|1-\frac{E_{\text {sim }}\left[T_{d}\right\rfloor}{E_{m}\left\lfloor T_{d}\right]}\right|(\%)$ & 1 & 5 & 9 & 9 & 24 \\
\hline
\end{tabular}

(B)

Table 2: Expected delivery delay calculated from (1) and by simulations for the RD model ( $\mu=0.0001:(\mathrm{A})$ $N=20$, and $R / L$ decreases. (B) $R / L=0.5 \%$, and $N$ increases).

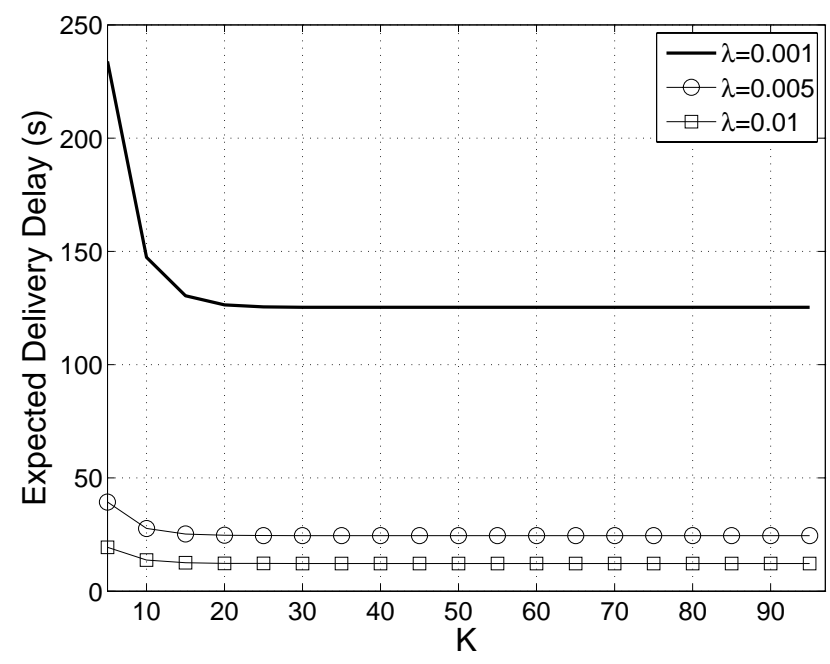

Figure 6: Expected delivery delay under $K$-limited two-hop relay protocol for different values of $K$ $(N=100, \mu=0.001)$.

form expressions have been derived for the probability distribution of the packet delivery delay, the expected number of copies in the system at the delivery instant, and the overall expected number of copies generated by the source at the delivery instant. We have observed that the latter metrics is directly related to the energy needed to transmit the packet to the destination node, in the case when the energy needed to transmit a packet between two nodes and the energy needed to decode a packet are constant. We have also proposed, and evaluated, a modification of the two-hop relay protocol that limits the number of copies of the packet that the source may generate.

In this paper our work has focused on the performance of the two-hop relay protocol before the destination receives the packet for the first time. It would also be interesting to quantify the impact of using an anti-packet mechanism on the total amount of energy consumed by the network during the entire lifetime of the packet, including its copies, in the network. Also, we have assumed that there is no timeout on the packet lifetime at the source. This assumption may not be realistic in some applications, and would therefore be worthwhile to relax it.

This study is part of a research effort towards developing simple analytical models for quantifying the performance of relay protocols for MANETs and, in particular, for better understanding the delay-energy tradeoff of this class of protocols.

\section{Appendix I: The fundamental matrix}

Lemma 1. The matrix $\mathbf{I}-\mathbf{Q}$ has $N$ distinct, real, and strictly negative eigenvalues $z_{1}, \ldots, z_{N}$ given by

$$
z_{k}=\frac{-N(2 \rho+1)+1-(N+1-2 k) \sqrt{4 \rho+1}}{2},
$$

for $k=1,2, \ldots, N$. Therefore the fundamental matrix $\mathbf{M}=$ $(\mathbf{I}-\mathbf{Q})^{-1}$ exists, and its (i,j)-entry is given by

$$
m(i, j)=-\frac{N \rho+j-1}{\left(\begin{array}{c}
N-1 \\
i-1
\end{array}\right) \rho^{i-1}} \sum_{k=1}^{N} \frac{\Psi_{i}^{k} \Psi_{j}^{k}}{z_{k} \Psi^{k} \tau^{2}\left(\Psi^{k}\right)^{T}},
$$

with $\Psi^{k}=\left(\Psi_{1}^{k}, \ldots, \Psi_{N}^{k}\right)$ where

$$
\begin{aligned}
\Psi_{i}^{k} & =(-1)^{i-1} x_{2}^{N-i} \sum_{l=l_{0}}^{l_{1}}\left(\begin{array}{c}
k-1 \\
l
\end{array}\right)\left(\begin{array}{c}
N-k \\
i-1-l
\end{array}\right)\left(\frac{x_{1}}{x_{2}}\right)^{k-1-l}, \\
x_{1} & =\frac{-1-\sqrt{1+4 \rho}}{2 \rho}, \quad x_{2}=\frac{-1+\sqrt{1+4 \rho}}{2 \rho}
\end{aligned}
$$

and where $l_{0}=\max (0, i-1-N+k), l_{1}=\min (i-1, k-1)$, and $\tau=\operatorname{diag}\left(\tau_{1}, \ldots, \tau_{N}\right)$, with $\tau_{i}=\left(\left(\begin{array}{c}N-1 \\ i-1\end{array}\right) \rho^{i-1}\right)^{-1 / 2} \cdot \diamond$

Proof. To simplify the computation of $\mathbf{M}$ we introduce the matrix A defined as

$$
\mathbf{A}=-\mathbf{B}(\mathbf{I}-\mathbf{Q})
$$

where $\mathbf{B}=\operatorname{diag}(b(1), \ldots, b(N))$ with $b(i)=N \rho+(i-1)$. Matrices $\mathbf{M}$ are $\mathbf{A}$ are related through the simple identity $\mathbf{M}=-\mathbf{A}^{-1} \mathbf{B}$. In the following we will compute $\mathbf{A}^{-1}$. We will follow the approach developed in [2]. We first compute the eigenvalues and left/right eigenvectors of $\mathbf{A}$.

Eigenvalues of $\mathbf{A}$.

Let $z$ be some eigenvalue of $\mathbf{A}$ and let $\boldsymbol{\Psi}=\left(\Psi_{1}, \ldots, \Psi_{N}\right)$ be the associated left eigenvector. That is, $\mathbf{\Psi} \mathbf{A}=z \mathbf{\Psi}$, or equivalently,

$$
\rho(N-(i-1)) \Psi_{i-1}-(\rho N+i-1+z) \Psi_{i}+i \Psi_{i+1}=0
$$

for $i=1, \ldots, N$, with $\Psi_{0}=\Psi_{N+1}=0$ by convention. Let $\psi(x)=\sum_{j=1}^{N} \Psi_{j} x^{j}$ denote the generating function of $\Psi$. Multiplying (18) by $x^{i}$ and then summing over $i$ yields

$$
\frac{\psi^{\prime}(x)}{\psi(x)}=\frac{\rho N x-(\rho N-1+z)-1 / x}{\rho x^{2}+x-1} .
$$

Let the zeros of $x^{2}+x / \rho-1 / \rho$ be $x_{1}=\frac{-1-\sqrt{1+4 \rho}}{2 \rho}$ and $x_{2}=$ $\frac{-1+\sqrt{1+4 \rho}}{2 \rho}$. The unique solution of (19) such that $\Psi_{N}=1$ is

$$
\begin{gathered}
\psi(x)=x\left(x_{1}-x\right)^{c_{1}}\left(x_{2}-x\right)^{c_{2}} \\
c_{1}:=\frac{x_{1}^{2} \rho N-x_{1}(\rho N-1+z)-1}{\rho x_{1}\left(x_{1}-x_{2}\right)}, \\
c_{2}:=\frac{-x_{2}^{2} \rho N+x_{2}(\rho N-1+z)+1}{\rho x_{2}\left(x_{1}-x_{2}\right)} .
\end{gathered}
$$


It is easily seen that $c_{1}+c_{2}=N-1$ (Hint: use $\left.x_{1} x_{2}=-1 / \rho\right)$, so that (20) also writes

$$
\psi(x)=x\left(x_{1}-x\right)^{c_{1}}\left(x_{2}-x\right)^{N-1-c_{1}} .
$$

Because $\psi(x)$ is a polynomial of degree $N$, we observe from (21) that necessarily $c_{1}$ is an integer lying in the set $\{0,1, \ldots$, $N-1\}$ since $x_{1}$ and $x_{2}$ are always distinct.

The equations $c_{1}=k-1$ for $k=1, \ldots, N$ give the following $N$ eigenvalues of $\mathbf{A}$ :

$$
z_{k}=\frac{-N(2 \rho+1)+1-(N+1-2 k) \sqrt{4 \rho+1}}{2}
$$

for $k=1, \ldots, N$. All eigenvalues of $\mathbf{A}$ are distinct (obvious from (22)). Furthermore, $z_{k}$ increases as $k$ increases, and it is easily seen that $z_{N}<0$ for $\rho>0$. Thus, $z_{k}<0$ for all $k=1, \cdots, N$.

Left eigenvectors of $\mathbf{A}$.

Recall that $\boldsymbol{\Psi}^{k}=\left(\Psi_{1}^{k}, \ldots, \Psi_{N}^{k}\right)$ is the left eigenvector associated with the eigenvalue $z_{k}$ of $\mathbf{A}$. The $i$ th component $\Psi_{i}^{k}$ of the eigenvector $\Psi^{k}$ is the coefficient of $x^{i}$ in the polynomial $x\left(x_{1}-x\right)^{k-1}\left(x_{2}-x\right)^{N-k}$ that is

$$
\Psi_{i}^{k}=(-1)^{i-1} x_{2}^{N-i} \sum_{l=l_{0}}^{l_{1}}\left(\begin{array}{c}
k-1 \\
l
\end{array}\right)\left(\begin{array}{c}
N-k \\
i-1-l
\end{array}\right)\left(\frac{x_{1}}{x_{2}}\right)^{k-1-l} .
$$

where $l_{0}=\max (0, i-1-N+k)$ and $l_{1}=\min (i-1, k-1)$.

Right eigenvectors of $\mathbf{A}$.

Recall that $\boldsymbol{\Phi}^{k}=\left(\Phi_{1}^{k}, \ldots, \Phi_{N}^{k}\right)^{T}$ is the right eigenvector associated with the eigenvalue $z_{k}$, for $k=1, \ldots, N$. We proceed like in [2, Section 2.4], that is we look for a diagonal matrix $\tau=\operatorname{diag}\left(\tau_{1}, \ldots, \tau_{N}\right)$ such that

$$
\tau^{-1} \mathbf{A} \tau=\left(\tau^{-1} \mathbf{A} \tau\right)^{T}
$$

It is easily found that (Hint: solve $\tau_{i}^{2} / \tau_{i+1}^{2}=\rho(N-i) / i$ for $i=1, \ldots, N$ with $\left.\tau_{1}=1\right)$

$$
\tau_{i}=\left(\left(\begin{array}{c}
N \\
i
\end{array}\right) \frac{i}{N} \rho^{i-1}\right)^{-1 / 2}, \quad i=1, \ldots, N
$$

satisfy (23). The identity $\boldsymbol{\Psi}^{k} \mathbf{A}=z_{k} \boldsymbol{\Psi}^{k}$ implies that

$$
\mathbf{\Psi}^{k} \tau\left(\tau^{-1} \mathbf{A} \tau\right)=z_{k} \boldsymbol{\Psi}^{k} \tau
$$

Therefore, $\mathbf{\Psi}^{k} \tau$ is a left eigenvector of the matrix $\tau^{-1} \mathbf{A} \tau$ associated with the eigenvalue $z_{k}$. Since the matrix $\tau^{-1} \mathbf{A} \tau$ is symmetric, it has identical left and right eigenvectors. Hence, $\left(\tau^{-1} \mathbf{A} \tau\right)\left(\boldsymbol{\Psi}^{k} \tau\right)^{T}=z_{k}\left(\boldsymbol{\Psi}^{k} \tau\right)^{T}$ which gives that

$$
\mathbf{A} \tau^{2}\left(\Psi^{k}\right)^{T}=z_{k} \tau^{2}\left(\Psi^{k}\right)^{T}
$$

This shows that $\alpha_{k} \tau^{2}\left(\Psi^{k}\right)^{T}$ is a right eigenvector associated with the eigenvalue $z_{k}$ for any constant $\alpha_{k} \neq 0$.

Without loss of generality we select the constants $\alpha_{1}, \cdots$ ,$\alpha_{N}$ so that $\boldsymbol{\Psi}^{k} \boldsymbol{\Phi}^{k}=1$ for every $k=1, \cdots, N$. Hence, $\alpha_{k}=1 / \Psi^{k} \tau^{2}\left(\Psi^{k}\right)^{T}$ for $k=1, \ldots, N$. Finally,

$$
\boldsymbol{\Phi}^{k}=\tau^{2}\left(\boldsymbol{\Psi}^{k}\right)^{T} / \Psi^{k} \tau^{2}\left(\boldsymbol{\Psi}^{k}\right)^{T},
$$

or equivalently

$$
\Phi_{i}^{k}=\frac{1}{\Psi^{k} \tau^{2}\left(\Psi^{k}\right)^{T}}\left(\left(\begin{array}{c}
N \\
i
\end{array}\right) \frac{i}{N} \rho^{i-1}\right)^{-1} \Psi_{i}^{k}, \quad i=1, \ldots, N .
$$

The proof is concluded by noting that

$$
\hat{a}(i, j)=\sum_{k=1}^{N} \frac{\Phi_{i}^{k} \Psi_{j}^{k}}{z_{k}}=\frac{1}{\left(\begin{array}{c}
N-1 \\
i-1
\end{array}\right) \rho^{i-1}} \sum_{k=1}^{N} \frac{\Psi_{i}^{k} \Psi_{j}^{k}}{z_{k} \boldsymbol{\Psi}^{k} \tau^{2}\left(\boldsymbol{\Psi}^{k}\right)^{T}},
$$

by using (24). Equation 25 together with $m(i, j)=-(N \rho+$ $(j-1)) \hat{a}(i, j)$ (coming from $\mathbf{M}=-\mathbf{A}^{-1} \mathbf{B}$ ) gives (16).

REMARK 7.1. Replacing $x$ by 1 in (19) implies the following relation between the eigenvalue $z_{k}$ and its corresponding left eigenvector $\boldsymbol{\Psi}^{\mathbf{k}}$

$$
\sum_{j=1}^{N} j \Psi_{j}^{k}=-\frac{z_{k}}{\rho} \sum_{j=1}^{N} \Psi_{j}^{k}, \quad 1 \leq k \leq N
$$

\section{Appendix II: Distribution of delivery delay}

The delivery delay, $T_{d}$, given that there are $i$ copies in the network at time 0 is the time to absorption of the Markov chain $\mathbf{M C}$ of Figure 1, given that $I(0)=i$. In order to compute the distribution of $T_{d}, P_{i}\left(T_{d} \leq t\right)$, given that $I(0)=$ $i$, first we derive the Laplace Steiltjes Transform (LST) given that $I(0)=i, f_{i}(s)=E_{i}\left[e^{-s T_{d}}\right]$, and next we invert $f_{i}(s)$. $f_{i}(s)$ will actually hold for any complex number $s$ such that $\Re(s) \geq 0$. Starting at state $i$, if we condition on the next possible transition of the $\mathbf{M C}, f_{i}(s)$ reads

$$
\begin{aligned}
f_{i}(s)= & \frac{\lambda i}{\lambda N+\mu(i-1)+s}+\frac{\mu(i-1)}{\lambda N+\mu(i-1)+s} f_{i-1}(s) \\
& +\frac{\lambda(N-i)}{\lambda N+\mu(i-1)+s} f_{i+1}(s)
\end{aligned}
$$

for $i=1, \ldots, N$ (by convention $f_{0}(\cdot)=f_{N+1}(\cdot)=1$ ). Multiplying both sides of $(27)$ by $\lambda N+\mu(i-1)+s$ and dividing by $\mu$, yields

$$
\begin{aligned}
(i-1) f_{i-1}(s) & -\left(\rho N+i-1+\frac{s}{\mu}\right) f_{i}(s) \\
& +\rho(N-i) f_{i+1}(s)=-\rho i
\end{aligned}
$$

for $i=1, \ldots, N$, with $\rho:=\lambda / \mu$. Let $\mathbf{f}=\left(f_{1}(s), \ldots, f_{N}(s)\right)^{T}$. In matrix form (28) writes

$$
\left(\mathbf{A}-\frac{s}{\mu} \mathbf{I}\right) \mathbf{f}=\mathbf{b}, \quad s \geq 0
$$

with I the $N$-by- $N$ identity matrix,

$$
\mathbf{b}:=(-\rho,-2 \rho, \cdots,-\rho N)^{T},
$$

and $\mathbf{A}$ is the N-by-N matrix defined in (17) in Appendix I. It is shown in Appendix I that the matrix $\mathbf{A}$ is invertible and diagonalizable, namely, there exists an invertible matrix $\mathbf{F}$ such that

$$
\mathbf{A}=\mathbf{F} \operatorname{diag}\left(z_{1}, \cdots, z_{N}\right) \mathbf{F}^{-1}
$$

where $z_{1}, \cdots, z_{N}$ are the eigenvalues of $\mathbf{A}$, the jth right eigenvector of $\mathbf{A}, \boldsymbol{\Phi}^{j}$, is the $j$ th column of the matrix $\mathbf{F}$, and the left eigenvector, $\boldsymbol{\Psi}^{i}$, is the $i$ th row of the matrix $\mathbf{F}^{-1}$. Hence

$$
\left(\mathbf{A}-\frac{s}{\mu} \mathbf{I}\right)^{-1}=\mathbf{F} \operatorname{diag}\left(\frac{1}{z_{1}-s / \mu}, \cdots, \frac{1}{z_{N}-s / \mu}\right) \mathbf{F}^{-1}
$$

provided that $z_{k}-s / \mu \neq 0$ for $k=1, \cdots, N$. Since, we know from Appendix I, that the eigenvalues of $\mathbf{A}$ are all strictly negative, so that the right-hand side of (30) is well defined (in particular) for all $s \geq 0$. Therefore for $s>0$, (cf. (29))

$$
\mathbf{f}=\mathbf{F} \operatorname{diag}\left(\frac{1}{z_{1}-s / \mu}, \cdots, \frac{1}{z_{N}-s / \mu}\right) \mathbf{F}^{-1} \mathbf{b}
$$


Since $\boldsymbol{\Phi}^{j}$ is the $j$ th column of the matrix $\mathbf{F}$, and $\boldsymbol{\Psi}^{i}$ is the $i$ th row of the matrix $\mathbf{F}^{-1}$, we see from (31) that the $i$ th component, $f_{i}(s)$, of the vector $\mathbf{f}$ is given by

$$
f_{i}(s)=-\rho \sum_{j=1}^{N} \sum_{k=1}^{N} \frac{j \Phi_{i}^{k} \Psi_{j}^{k}}{z_{k}-s / \mu}, \quad s \geq 0 .
$$

Closed-form expressions for the eigenvalues and right/left eigenvectors of the matrix $\mathbf{A}$ are provided in Lemma 1. Remark 7.1 and Lemma 1 of Appendix I give that

$$
f_{i}(s)=\frac{1}{\left(\left(\begin{array}{c}
N \\
i
\end{array}\right) \frac{i}{N} \rho^{i-1}\right)} \sum_{k=1}^{N} \frac{\Psi_{i}^{k}\left(\boldsymbol{\Psi}^{k} \mathbf{1}^{T}\right)}{\boldsymbol{\Psi}^{k} \tau^{2}\left(\mathbf{\Psi}^{k}\right)^{T}} \frac{z_{k}}{z_{k}-s / \mu},
$$

for $s \geq 0$, where $\mathbf{1}^{T}$ is the column vector of dimension $N$ whose all components are equal to 1, one. Equation $33 \mathrm{im}-$ plies that the distribution of $T_{d}$ given that the $\mathbf{M C}$ starts at state 1 reads

$$
\begin{gathered}
P_{i}\left(T_{d} \geq t\right)=\frac{1}{\left(\begin{array}{c}
N-1 \\
i-1
\end{array}\right) \rho^{i-1}} \sum_{k=1}^{N} \frac{\Psi_{i}^{k}}{\boldsymbol{\Psi}^{k} \tau^{2}\left(\mathbf{\Psi}^{k}\right)^{T}} \mathbf{\Psi}^{k} \mathbf{1}^{T} e^{z_{k} \mu t} \\
\text { Since } z_{k}<0 \text { for } 1 \leq k \leq N \text { and } \rho>0 \text {, thus } \\
\lim _{t \rightarrow+\infty} P_{i}\left(T_{d}>t\right)=0 .
\end{gathered}
$$

Further, $P_{i}\left(T_{d}>0\right)=1$ (Hint: $\Psi^{\mathbf{i}} \Phi^{\mathbf{j}}=0$ for $i \neq j$, and $\left.\boldsymbol{\Psi}^{\mathrm{k}} \boldsymbol{\Phi}^{\mathrm{k}}=1\right)$.

\section{REFERENCES}

[1] Delay tolerant research group. Web site: http://www.dtnrg.org.

[2] D. Anick, D. Mitra, and M. M. Sondhi. Stochastic theory of a data-handling system with multiple sources. Bell System Technical Journal, 61(8):1871-1896, Oct. 1982.

[3] C. Bettstetter. Mobility modeling in wireless networks: Categorization, smooth movement, border effects. ACM Mobile Computing and Communications Review, 5(3):55-67, July 2001.

[4] J.-Y. L. Boudec and M. Vojnovic. Perfect simulation and stationarity of a class of mobility models. In Proc. of IEEE INFOCOM, Miami, FL, Apr. 2005.

[5] J. Broch, A. D. Maltz, B. D. Johnson, Y.-C.Hu, and Jetcheva. A performance comparison of multi-hop wireless ad hoc network routing protocols. In Proc. of ACM MOBICOM, pages 85-97, Dallas, TX, Oct. 1998.

[6] A. E. Gamal, J. Mammen, B. Prabhakar, and D. Shah. Throughput-delay trade-off in wireless networks. In Proc. of IEEE INFOCOM, Hong Kong, Apr. 2004.

[7] C. Grinstead and J. Snell. Introduction to Probability. American Mathematical Society, 1997.

[8] R. Groenevelt, P. Nain, and G. Koole. The message delay in mobile ad hoc networks. In Posters ACM SIGMETRICS 2005, Canada, 2005.

[9] M. Grossglauser and D. Tse. Mobility increases the capacity of ad hoc wireless networks. ACM/IEEE Transactions on Networking, 10(4):477-486, Aug. 2002.

[10] P. Gupta and P. R. Kumar. The capacity of wireless networks. ACM/IEEE Transactions on Information Theory, 46(2), Mar. 2000.
[11] A. A. Hanbali, P. Nain, and E. Altman. Performance evaluation of packet relaying in ad hoc network. Technical Report RR-5860, INRIA, Mar. 2006.

[12] T. G. Kurtz. Solutions of ordinary differential equations as limits of pure jump markov processes. Applied Probility, 7:49-58, 1970.

[13] B. L. P. Nain, D. Towsley and Z. Liu. Properties of random direction models. In Proc. of IEEE INFOCOM, Miami, FL, Mar. 2005.

[14] T. Small and Z. J. Haas. The shared wireless infostation model: A new ad hoc networking paradigm. In Proc. of ACM MOBIHOC, Anapolis, MD, USA, 2003.

[15] T. Small and Z. J. Haas. Resource and performance tradeoffs in delay-tolerant wireless networks. In Proc. ACM SIGCOM Workshop on Delay-Tolerant Networks, Philadelphia, PA, USA, Aug. 2005.

[16] E. Zhang, G. Neglia, J. Kurose, and D. Towsley. Performance modeling of epidemic routing. In Proc. of Networking, pages 827-839, Coimbra, Portugal, May 2006 . 